\title{
The tibialis anterior reflex in healthy subjects and in L5 radicular compression
}

\author{
J STAM \\ From the Department of Neurology, Academic Medical Centre, University of Amsterdam, Amsterdam, The \\ Netherlands
}

SUMMARY Phasic stretch reflexes were evoked in the tibialis anterior (TA) muscle, by tapping the dorsal side of the foot with a hand-held reflex hammer. The responses were recorded by means of surface electrodes. The TA refiex was examined in 70 healthy subjects and in 18 patients with L5 radicular compression. In $58(83 \%)$ of the healthy subjects the reflex could be recorded bilaterally, in eight (11\%) subjects no reflex was found on either side, and in four (6\%) it was absent in one leg. Simultaneous recordings from the gastrocnemius-soleus showed that TA responses were not caused by volume conduction from that muscle. In the 18 patients with L5 radicular compression the TA reflex was absent on the affected side 13 times $(72 \%)$ and present bilaterally in the other five cases. If asymmetry of the reflex (unilateral absence) is considered as a test for the presence of L5 radicular compression, the likelihood ratio for a positive test is $12 \cdot 0$, and for a negative test $0 \cdot 3$. The examination of the TA reflex is easily performed and can be useful in the diagnosis of L5 radicular compression.

The history and neurological examination are often sufficiently reliable for the diagnosis of lumbar radicular syndromes, especially in the presence of objective signs such as weakness, wasting, and reflex abnormalities. In the absence of weakness, or if weakness is difficult to assess, diminution or absence of the knee or ankle jerks are especially valuable signs and help to establish the segmental level of root compression. Unfortunately, the muscles which are mainly innervated by the L5 root usually do not show a visible response to phasic stretch. If present, these reflexes are small and inconsistent. Reflexes in the tibialis posterior, ${ }^{12}$ the extensor hallucis longus ${ }^{3}$ and the peroneal muscles ${ }^{4}$ can be observed in patients with brisk reflexes, but in most cases their diagnostic value is limited. In healthy subjects usually no phasic stretch reflex in the tibialis anterior muscle can be seen on bed-side examination, but a short latency electromyographical response has been recorded in that muscle after phasic stretch. ${ }^{56}$

We examined the possibility of recording phasic stretch reflexes in the tibialis anterior muscle with a

Address for reprint requests: Dr J Stam, Department of Neurology, Academic Medical Centre, 1105 AZ Amsterdam, The Netherlands.

Received 14 August 1987.

Accepted 22 September 1987 simple method in healthy subjects, and studied the diagnostic value of this method in patients with L5 radicular compression syndromes.

\section{Methods}

Phasic stretch reflexes in the tibialis anterior (TA) muscle were evoked by tapping the dorsal and distal part of the first metatarsal bone with a hand-held reflex hammer, provided with a piëzo-electric transducer which delivers a trigger pulse and a signal for monitoring the strength of the tap. ${ }^{7}$ The subjects were in supine position and the taps were directed caudally to cause a brief stretch of the foot dorsiflexors. A wooden spatula was pressed against the first metatarsal and served to receive the taps. Direct tapping of the TA tendon was tried in nine subjects but proved unsatisfactory.

The reflex responses were recorded with bipolar disposable surface electrodes $\left(\mathrm{Ag}-\mathrm{AgCl}, 0.6 \mathrm{~cm}^{2}\right)$ placed $5 \mathrm{~cm}$ apart, on either side of the centre of the tibialis anterior muscle and $2 \mathrm{~cm}$ laterally from the anterior margin of the tibia. When no response could be recorded from the relaxed TA, subjects were instructed to maintain slight isometric dorsiflexion of the foot by lightly pressing against the spatula. In those cases the responses were averaged to enhance the contrast between reflex response and background activity. In most subjects a reproducible response was present after averaging 16 times. When no reproducible response was visible after averaging 32 times the reflex was judged to be absent.

The latencies of the tibialis anterior reflexes were mea- 
sured from the trigger pulse generated by the tap to the first deflection from the base-line due to the reflex potential. The reflex amplitude was defined as the peak-to-peak amplitude in millivolts.

Simultaneous recordings from the gastrocnemius-soleus and TA muscles after phasic stretch of the TA with the described technique were made in 15 subjects to examine the possible influence of volume conduction.

The TA reflex was examined bilaterally in 70 consecutive healthy subjects aged 19 to 36 years (mean 25). In 34 of these subjects the mechanically evoked Achilles (soleus) reflex was also recorded on one side.

In 18 patients with L5 radicular compression syndromes, aged 20 to 60 (mean 37) the TA reflexes were recorded bilaterally. The diagnosis was based upon the presence of a typical history of low back pain with irradiating pain which became worse during straight leg raising, in combination with the following signs (table 1):

(1) Weakness of the foot dorsiflexors alone (seven patients)

(2) (1) and a diminished ankle jerk (three patients)

(3) (1) and a diminished knee jerk (two patients)

(4) (1) and (2) and (3) (one patient)

(5) No weakness or reflex abnormalities (five patients).

The last group was included because it is well known that a clinical presentation with few clinical signs may be caused by a L5 radicular syndrome. In all patients the diagnosis of L5 radicular compression was made by a neurologist, independently of the results of TA-reflex recording.

Sensory abnormalities (except pain) in the L5 dermatome were found in five patients. In 10 cases the diagnosis was confirmed by radiological demonstration of L5 root compression by an intervertebral disc protrusion (myelography in nine and computed tomography in one). A laminectomy was performed in five of these patients and surgical findings confirmed the clinical and radiological diagnosis. In two patients the myelogram was normal but the clinical diagnosis of L5 radicular compression was maintained, and in one of them confirmed by needle EMG. In the remaining six patients only plain radiographs of the lumbar spine were made. In 11 patients routine EMG studies were performed. In nine patients the results were consistent with the diagnosis of L5 compression.

\section{Data analysis}

The unilateral absence of tibialis anterior reflexes was compared in patients and healthy controls by Fisher's exact test and by calculating sensitivity, specificity and likelihood ratios (the likelihood ratio for a positive test result gives the odds that that result comes from a patient with L5 compression $^{8}$ ). TA amplitudes and latencies, and the leftright asymmetry of these measures, were compared in patients and controls with the Mann-Whitney U-test. The relation between latencies of the TA and soleus reflexes to subject length and age was examined with linear regression analysis. The relation between TA-paresis and TA-reflex asymmetry was examined with the Mann-Whitney U-test.

\section{Results}

\section{Healthy subjects}

In $58(83 \%)$ of the 70 examined subjects bilateral reflexes in the tibialis anterior muscle were present. In eight $(11 \%)$ subjects no reflexes could be recorded on both sides, and in four (6\%) subjects the reflex was absent unilaterally (table 2). Typical recordings are shown in fig 1 . The mean amplitude of the TA reflex was $0.15 \mathrm{mV}$ (SD 0.11, range 0.03-0.61), which is about 30 times smaller than the average amplitude of maximal soleus reflexes in healthy subjects. Isometrie contraction of the TA and averaging were usuall $B$ necessary to record the reflex. The right- and lefto sided amplitudes were significantly correlated $(\mathrm{R}=0.77, \mathrm{p}<0.001)$ but some degree of asymmetry in amplitudes was found in many subjects. The aver-

Table 1 Clinical and paraclinical data and results of $T A$ reflex examination

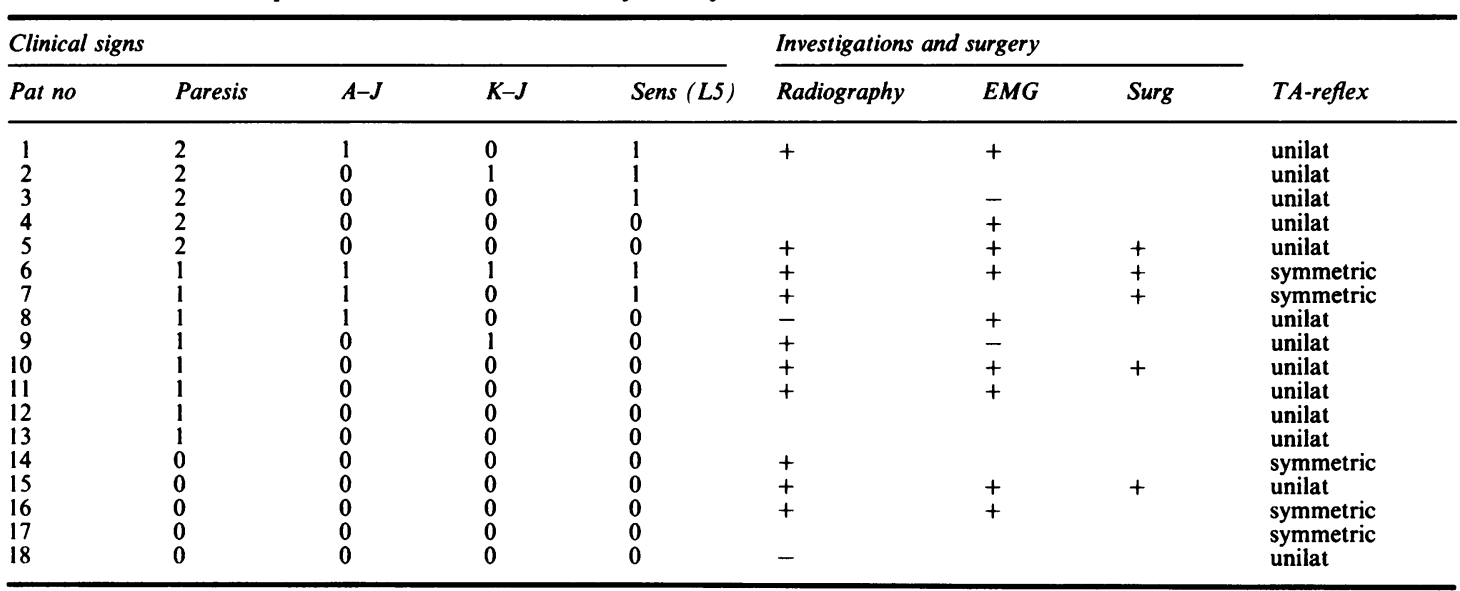

Clinical signs: Paresis: $2=$ moderate, $1=$ slight, $0=$ absent. $A-J=$ ankle jerk, $\mathrm{K}-\mathrm{J}=$ knee-jerk: $1=$ diminished, $0=$ normal. Sens $=$ sensory abnormality in L5 dermatome (except pain): $1=$ present, $0=$ absent. Examinations and surgery: $+=$ consistent with L5 lesion, $-=$ normal, blank = not performed. TA-reflex: unilat $=$ unilaterally absent. 
age left-right difference (absolute value) was $0.05 \mathrm{mV}$ (SD 0.05 , range 0.00 to 0.30 , table 2 ).

Although the described method of tapping the foot causes stretch of the TA, spreading of the mechanical vibration may cause spindle activation in remote muscles such as the soleus and gastrocnemius, and subsequently evoke reflexes in those muscles ("reflex irradiation"9). Such reflex activity in remote muscles may be picked up by the electrodes over the TA. This possibility was examined in 15 subjects by recording simultaneously from the TA and the soleus during tapping the first metatarsal. A signal from the electrodes over the soleus could be recorded in a number of subjects in this way, but the amplitude of this signal was always less then the amplitude of the potential recorded over the TA (fig 2). If the responses recorded over the TA were caused by volume conduction from the antagonists one would expect to find the larger amplitudes in the soleus.

The latencies of the tibialis anterior reflexes showed a linear relation with the height of subjects, with correlation coefficients of 0.73 and 0.70 (left and right respectively, $p<0.001$, table 2 ). The TA latencies were on average $3.5 \mathrm{~ms}$ longer than the soleus reflex latencies (fig 3). There was no significant relation between TA latency and age ( $R 0.03, p>0.75)$. The mean difference between right and left sided TA reflex latency (absolute value) was $1.3 \mathrm{~ms}$ (SD $1 \cdot 1$, range 0.0 to $4.0 \mathrm{~ms}$ ).

\section{Patients}

In the 18 patients with L5 radicular compression syndromes the tibialis anterior reflex was unilaterally absent in the affected leg in 13 cases $(72 \%)$. In the remaining five patients the reflex was present on both sides. In the 10 patients with a radiologically confirmed L5 compression the TA reflex in the affected leg was absent six times. The frequency of one-sided absent reflexes in the patients was significantly greater than in the healthy controls (Fisher's test, $p<0.0001$ ). No relation between absence or presence of the TA reflex and some characteristics of the patients, such as age, abnormal knee or ankle jerks, results of radiological examinations, or need for surgical therapy could be demonstrated (table 1). The only factor related to absence of a TA reflex was weakness of the foot dorsiflexors. In all five patients with moderate weakness the TA reflexes were absent on the affected side. In the eight patients with slight paresis, six had unilaterally absent TA reflexes, and in the five patients without paresis the TA reflex was absent in two cases. Paresis was significantly more frequent in patients with asymmetric TA reflexes $(p=0.03$, Mann-Whitney U-test).

The amplitudes and latencies of positive (non-zero) TA reflexes in the patients did not differ significantly from those values in healthy controls (table 2). The left-right differences of the amplitudes and latencies in patients with bilateral TA reflexes (five cases) were

Table 2 Quantitative differences between $T A$ reflexes in controls and patients

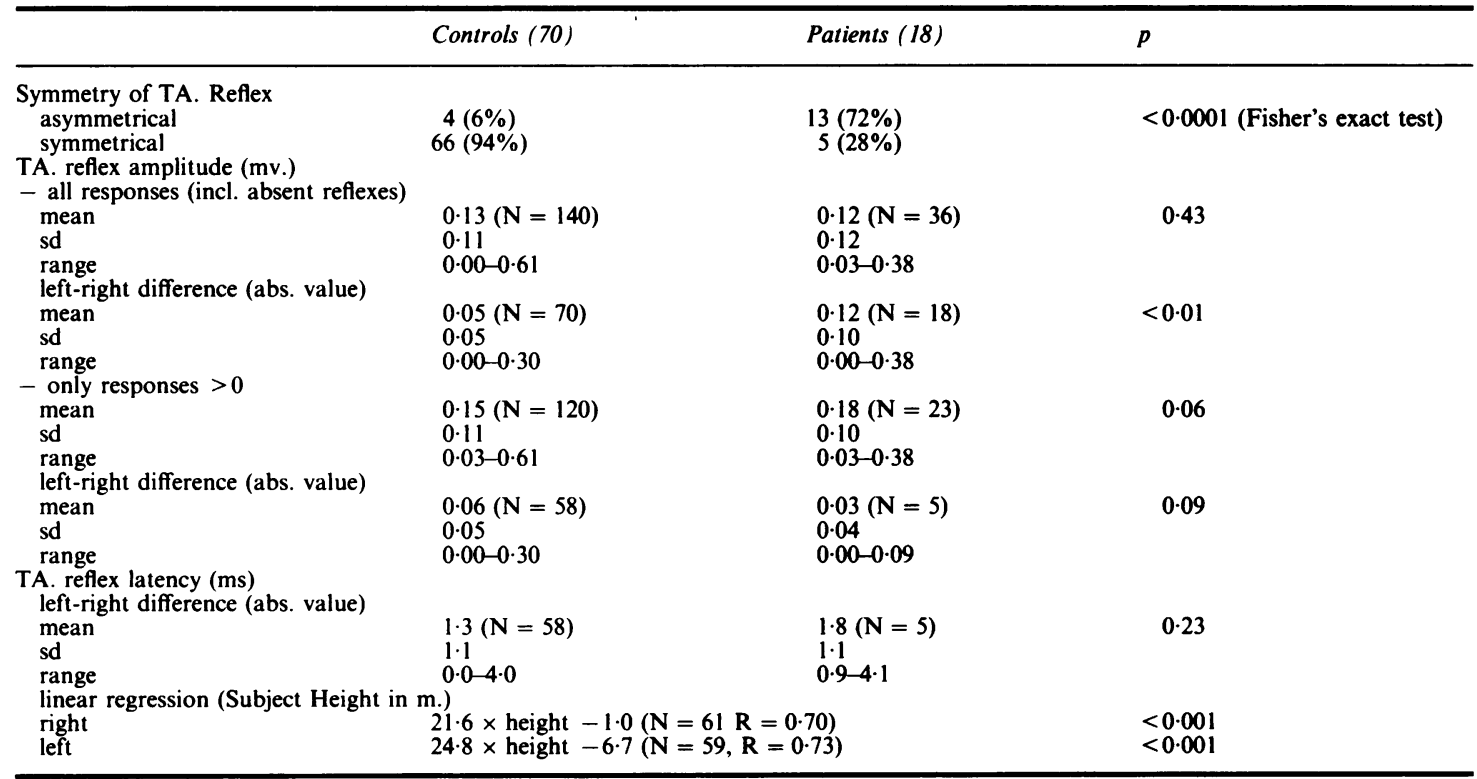

All $p$ values were obtained by applying the Mann-Whitney $U$ test except the values for symmetry of the TA reflex (Fisher's exact test) and for linear regression analysis (significance of correlation). 
<smiles>C#CCCCCCCCCCCCCCCCCO</smiles>

Left

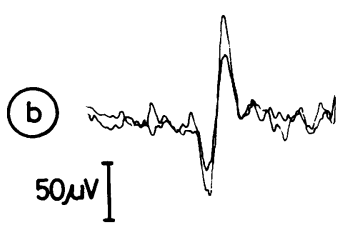

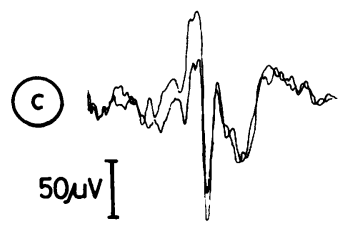

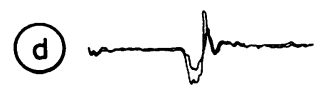

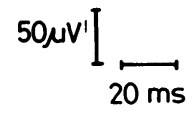
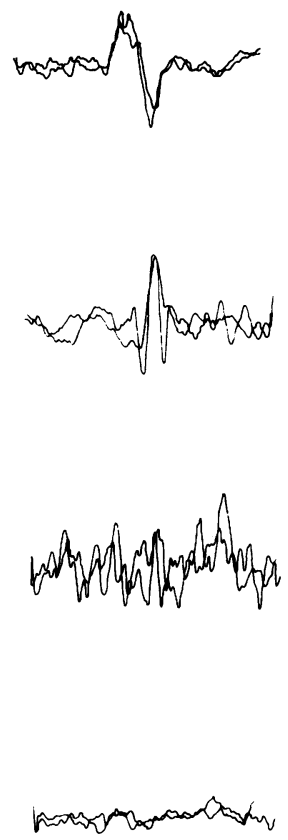

$20 \mathrm{~ms}$

Fig 1 Tibialis anterior reflexes recorded in two healthy subjects ( $a$ and $b$ ) and in two patients with left-sided L5 radicular compression ( $c$ and d). 16 Reflex responses were averaged for each record, and two superimposed records are shown for each reflex. In $a$ and $b$ reproducible reflexes are recorded on both sides. In $b$ and $c$ no response its present on the affected (left) side. Background EMG activity is seen in all records, also in the muscles without detectable TA reflex activity.

within the range for normal subjects, except in one patient with a difference in latency of $4.1 \mathrm{~ms}$. This was one of the two patients with one-sided weakness of the foot dorsiflexors and bilaterally positive TA reflexes.

From the frequencies of a unilaterally absent TA reflex in patients and healthy controls the following test characteristics can be calculated. Sensitivity 0.72 (95\% confidence interval $0.46-0.90)$, specificity 0.94 $(0.86$ to 0.98$)$, likelihood ratio for a positive test 12.0 , and likelihood ratio for a negative test $0 \cdot 3$. If the analysis is limited to the radiologically confirmed cases the sensitivity is 0.60 , and the specificity, which is based upon the findings in controls, remains 0.94 .

\section{Discussion}

With the described method we found TA reflexes in the majority of healthy subjects, which is what one would expect for a normal skeletal muscle. Compared with a previously described method of studying this reflex 5 the frequency of absent reflexes is small, which indicates that the dorsal foot tap as described is an effective way to stimulate the dynamic muscle spindles. Phasic stretch reflexes in the TA have been studied by plantar flexing the foot with a torque motor. ${ }^{510}$ In one study a TA response was observed 80-100 ms after the onset of plantar flexion and it can not be regarded as equivalent to the tendon reflex. ${ }^{10}$ Chan et al occasionally recorded a TA response in relaxed subjects, $40 \mathrm{~ms}$ after the onset of passive plantar flexion. ${ }^{5}$ In four out of nine subjects who resisted plantar flexion a small response in the TA was seen at that latency. This response is likely to be identical with the presently described reflexes. In healthy subjects and in patients with a spastic paresis a TA response can be found after tapping the Achilles tendon ("reciprocal excitation"11-13).

H-reflexes in the TA after stimulation of the peroneal nerve are usually absent or inconsistent. Delwaide $e t$ al recorded TA H-reflexes with an average amplitude of $0.10 \mathrm{mV}$ in seven out of 25 norma subjects. ${ }^{14}$ Garcia et al were able to record $\mathrm{H}$-reflexeso in the TA during contraction of the antagonists, but only after facilitation by double electrical stimuli to the peroneal nerve. ${ }^{15}$

From these studies and the present results we contō clude that taps with a reflex hammer as described are probably more effective stimuli for evoking TA

$$
\text { I }
$$

TA

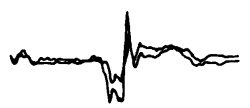

SOL

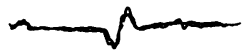

$\left.50 \mu \mathrm{V}\right|_{20 \mathrm{~ms}}$

Fig 2 Simultaneous recordings of the signal from the electrodes over the tibialis anterior (TA) and over the gastrocnemius-soleus (SOL) after tapping the dorsal side of the foot to induce phasic stretch of the TA. Examples in two healthy subjects show clear reflexes in the TA, and some simultaneous activity recorded over the SOL, indicating that the TA activity is not caused by crosstalk from the SOL. 

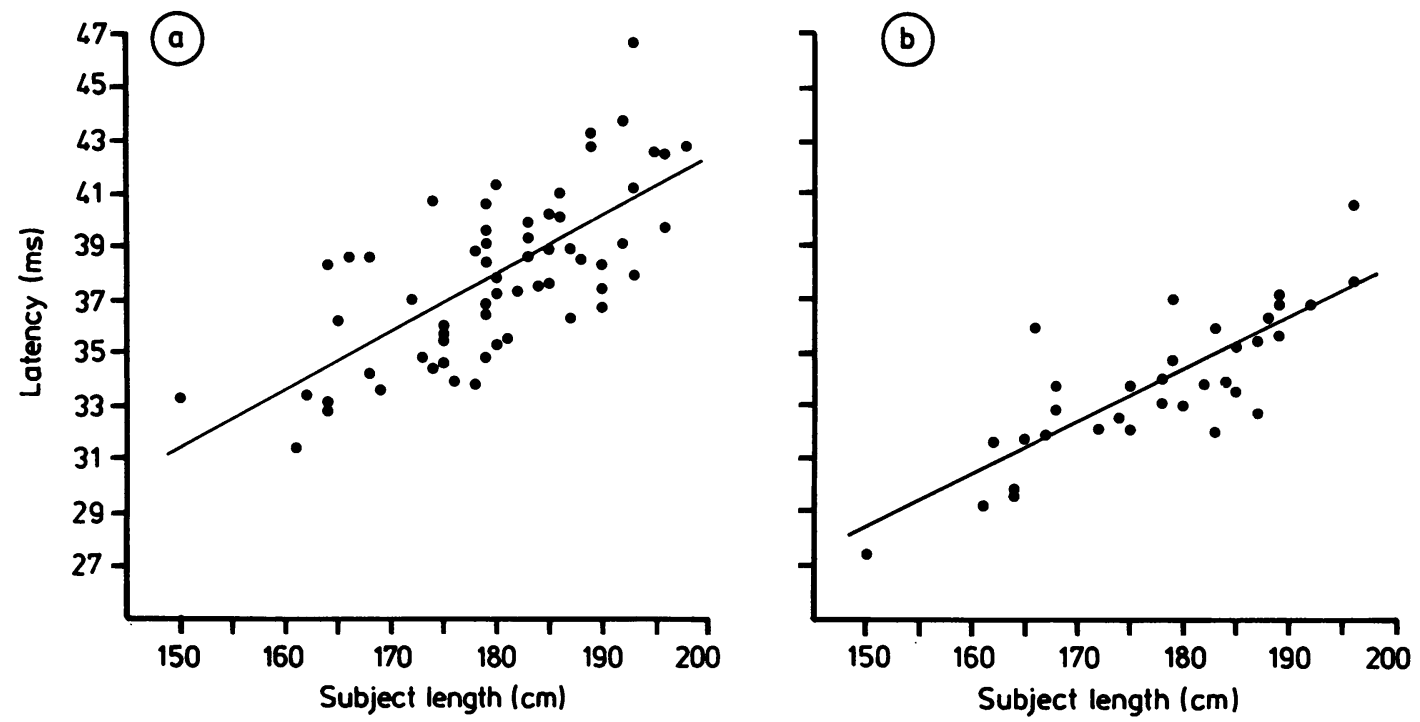

Fig 3 Latencies of the right-sided $T A$ reflexes $(a, N=61)$ and of the right-sided soleus reflexes $(b, N=34)$ in healthy subjects. Both latencies are significantly correlated with subject height $(R 0.70$ and 0.82 respectively, $p>0.001)$. The latencies of the TA reflex are larger than the latencies of the soleus reflex and show a larger residual variance.

reflexes than phasic stretch by torque motors or electrical stimulation of the peroneal nerve.

The reflex-amplitudes are small compared with the normal soleus reflex, and show considerable interand intra-individual variation. The finding of bilaterally absent TA reflexes in $11 \%$ of the healthy subjects is probably a manifestation of this normal variability.

Asymmetry of TA amplitudes was found frequently, in agreement with previous measurements of other reflexes in healthy subjects (for example, the knee jerk, ${ }^{16}$ and the soleus $\mathrm{H}-$ reflex $^{17}$ ). The fact that in $6 \%$ of the healthy subjects no TA reflex could be recorded on one side is probably another manifestation of this normal reflex-asymmetry. It might be possible to record TA reflexes with needle- or wireelectrodes in subjects who had absent TA reflexes with the described technique, but this was not examined in the present study.

Because of their small amplitudes and the necessity of isometric contraction and averaging, the latencies of the TA reflexes can be measured with less precision than soleus-reflex latencies. This fact probably causes the larger residual variance of the TA latencies after linear regression, and the larger left-right differences of the TA latencies (mean difference $1.3 \mathrm{~ms}$ ) compared with the soleus (mean $0.7 \mathrm{~ms}$ ). The fact that TA latencies are longer than soleus latencies cannot be explained by assuming a longer reflex arc of the TA: The L5 and L4 segments are more proximal than the S1 segment of the soleus reflex, but the TA muscle is also more proximal. The poor correlation between TA reflex latency and age can be explained by the lack of elderly subjects in our control group.

The TA reflexes in the patients we studied differed in only one aspect from the reflexes in healthy subjects: unilateral absence of the reflex was far more common in the patient group than in the control group. The amplitudes and latencies of the positive (non-zero) reflexes in the patients did not differ significantly from the values in healthy controls. This indicates that the larger number of unilaterally absent TA reflexes in the patients cannot be attributed to normal asymmetry in combination with general hyporeflexia, as might be possible in older subjects. The average amplitude of the positive TA reflexes was even larger in patients (though not significantly, $p=0.06$ ) than in healthy subjects. The pathological significance of unilateral absence of TA reflexes in the patients is further indicated by the fact that it never occurred in the unaffected leg.

Too strict selection of patients, for example by only admitting patients with abnormal myelograms, would have limited the practical applicability of the results. ${ }^{18}$ Therefore we applied clinical instead of radiological or surgical diagnostic criteria. This resulted in the inclusion of two patients with normal myelograms, one of whom showed an absent TA reflex on the affected side. The latter finding may be interpreted as a chance event $(6 \%$ of normal controls had one absent TA reflex), or as an example of a false 
negative myelography in which the TA reflex confirms the clinical diagnosis of L5 compression, for instance by entrapment in the lateral recess. ${ }^{19}$

The fact that the TA reflex was more often absent in patients with some degree of TA paresis is not surprising. It could be argued that this limits the practical usefulness of the TA reflex, because in those cases the TA reflex does not add much to the bedside examination. However, posterior root compression may interrupt the reflex arc without causing weakness. Analogous to clinical experience with the knee and ankle jerks, absent TA reflexes may likewise be expected to occur in the absence of paresis.

The sensitivity of unilateral absence of the TA reflex in this study is $72 \%$, and the $95 \%$ confidence interval is rather wide (46-90\%) owing to the small number of patients. Thus a number of normal (false negative) results is to be expected in practice. Yet the TA recording can give useful information, especially when it is abnormal, that is, absent on the affected side, as indicated by the likelihood ratio for a positive test of 12.0 . In a patient with an estimated a priori likelihood of L5 compression of $50 \%$ for instance, the finding of an abnormal TA reflex would change this likelihood to $92 \%$ based upon the present data. ${ }^{8}$

Most authors agree that EMG examinations, including $\mathrm{H}$-reflexes, have a complementary place in the examination of patients with possible lumbar radicular compression, although these techniques do not provide a "gold standard". ${ }^{20-22}$ It has further been shown that recording the soleus tendon reflex is superior to $\mathrm{H}$-reflex measurement for the diagnosis of S1 root lesions. ${ }^{23}$ We now add the mechanically evoked tibialis anterior reflex to the techniques which may increase the sensitivity of the EMG examination in $\mathrm{L} 5$ lesions.

In conclusion, the tibialis anterior reflex is easily recorded in most healthy subjects with a technique available in every EMG laboratory, and may be useful in the diagnosis of patients with suspected L5 radicular compression.

We thank Professor Dr H van Crevel and Dr BW Ongerboer de Visser for their support and valuable comments, NJD Nagelkerke for statistical advice, and GJ van Bruggen, JFM Mutsaerts and HB Fortuin for their assistance during the experiments.

\section{References}

1 Mumenthaler M, Schliack H. Läsionen Peripherer Nerven.
Thieme, Stuttgart, 1973:55.

2 DeJong RN. The Neurologic Examination. Harper \& Row, New York, 1979:440.

3 Taylor TKF, Wienir M. Great-toe extensor reflexes in the diagnosis of lumbar disc disorder. Br Med J 1969;2:487-9.

4 Berlin L. A peroneal muscle stretch reflex. Neurology 1971;21:1177-8.

5 Chan CWY, Kearney RE, Melvill Jones G. Tibialis anterior response to sudden ankle displacements in normal and Parkinsonian subjects. Brian Res 1979;173:303-14.

6 Iles JF. Responses in human pretibial muscles to sudden stretch and to nerve stimulation. Exp Brain Res 1977;30:451-70.

7 Stam J, van Leeuwen JR. A simple measurement hammer for quantitative reflex studies. Electroencephalogr Clin Neurophysiol 1984;58:282-4.

8 Feinstein AR. Clinical epidemiology; The architecture of Clinical Research. Philadelphia, Saunders, 1985:437.

9 Lance JW. The mechanism of reflex irradiation. Proc Aust Assoc Neurol 1965;3:77-81.

10 Gottlieb GL, Agarwal GC. Response to sudden torques about ankle in man: Myotatic reflex. J Neurophysiol 1979;42:91-106.

11 Rao VR. Reciprocal inhibition: Inapplicability to tendon jerks. $J$ Postgrad Med 1965;11:123-5.

12 Gottlieb GL, Myklebust BM, Penn RD, Agarwal GC. Reciprocal excitation of muscle antagonists by the primary afferent pathway. Exp Brain Res 1982;46:454-6.

13 Mykleburst BM, Gottlieb GL, Penn RD, Agarwal GC. Reciprocal excitation of antagonist muscles as a differentiating feature in spasticity. Ann Neurol 1982;12:367-74.

14 Delwaide PJ, Crenna P, Fleron MH. Cutaneous nerve stimu lation and motoneural excitability I: Soleus and tibialis anterior excitability after ipsilateral and contra-lateral sural nerve्ष stimulation. J Neurol Neurosurg Psychiatry 1981;44:699-707.@

15 Garcia HA, Fisher MA, Gilai A. H-reflex analysis of segmenta reflex excitability in flexor and extensor muscles. Neurolog. 1979;29:984-91.

16 Simons DG, Dimitrijevic MR. Quantitative variations in th8 force of quadriceps responses to ser patellar taps in normat man. Am J Phys Med 1972;51:240-63.

17 Hugon M. Methodology of the Hoffmann reflex in man. If Desmedt JE. New Developments in Electromyography and Clin ical Neurophysiology, Vol 3. Basel: Karger, 1973:277-93.

18 Ransohoff DF, Feinstein AR. Problems of spectrum and bias in evaluating the efficacy of diagnostic tests. $N$ Engl $J$ Med 1978;299:926-30.

19 Reynolds AF, Weinstein PR, Wachter RD. Lumbar monoradiculopathy due to unilateral facet hypertrophy. Neurosurgery 1982;10:480-6.

20 Tonzola RF, Ackil AA, Shahani BT, Young RR. Usefulness of electrophysiological studies in the diagnosis of lumbosacral root disease. Ann Neurol 1981;9:305-8.

21 Khatri BO, Baruah J, McQuillen MP. Correlation of electromyography with computed tomography in evaluation of lower back pain. Arch Neurol 1984;41:594-7.

22 Aminoff MJ, Goodin DS, Parry GJ, Barbaro NM, Weinstein PR, Rosenblum ML. Electrophysiologic evaluation of lumbosacral radiculopathies: Electromyography, late responses, and somatosensory evoked potentials. Neurology 1985;35:1514-8.

23 Rico RE, Jonkman EJ. Measurement of the Achilles tendon reflex for the diagnosis of lumbar root compression syndromes. J Neurol Neurosurg Psychiatry 1982;45:791-5. 